\title{
Five-torsion in the homology of the matching complex on 14 vertices
}

\author{
Jakob Jonsson
}

Received: 26 April 2007 / Accepted: 16 January 2008 / Published online: 5 February 2008

(C) Springer Science+Business Media, LLC 2008

\begin{abstract}
J.L. Andersen proved that there is 5-torsion in the bottom nonvanishing homology group of the simplicial complex of graphs of degree at most two on seven vertices. We use this result to demonstrate that there is 5-torsion also in the bottom nonvanishing homology group of the matching complex $\mathrm{M}_{14}$ on 14 vertices. Combining our observation with results due to Bouc and to Shareshian and Wachs, we conclude that the case $n=14$ is exceptional; for all other $n$, the torsion subgroup of the bottom nonvanishing homology group has exponent three or is zero. The possibility remains that there is other torsion than 3-torsion in higher-degree homology groups of $\mathrm{M}_{n}$ when $n \geq 13$ and $n \neq 14$.
\end{abstract}

Keywords Matching complex $\cdot$ Simplicial homology $\cdot$ Torsion subgroup

\section{Introduction}

Throughout this note, by a graph we mean a finite graph with loops allowed but with no multiple edges or multiple loops. The degree of a vertex $i$ in a given graph $G$ is the number of times $i$ appears as an endpoint of an edge in $G$; thus a loop at $i$ (if present) is counted twice, whereas other edges containing $i$ are counted once.

Given a family $\Delta$ of graphs on a fixed vertex set, we identify each member of $\Delta$ with its edge set. In particular, if $\Delta$ is closed under deletion of edges, then $\Delta$ is an abstract simplicial complex. Let $n \geq 1$ and let $\lambda=\left(\lambda_{1}, \ldots, \lambda_{n}\right)$ be a sequence of nonnegative integers. We define $\mathrm{BD}_{n}^{\lambda}$ to be the simplicial complex of graphs on the vertex set $[n]:=\{1, \ldots, n\}$ such that the degree of the vertex $i$ is at most $\lambda_{i}$. We write

Research of J. Jonsson was supported by European Graduate Program "Combinatorics, Geometry, and Computation", DFG-GRK 588/2.

J. Jonsson ( $\varangle)$

Dept. of Mathematics, KTH, Stockholm, 100 44, Sweden

e-mail: jakob_jonsson@yahoo.se 
$\mathrm{BD}_{n}^{k}:=\mathrm{BD}_{n}^{(k, \ldots, k)}$ and $\mathrm{M}_{n}:=\mathrm{BD}_{n}^{1}$; the latter complex is the matching complex on $n$ vertices.

The topology of $\mathrm{M}_{n}$ and related complexes has been subject to analysis in several theses $[1,7,10,12,13,15,17]$ and papers [2-5, 8, 9, 16, 19, 20, 22]; see Wachs [21] for an excellent survey and further references.

The prime 3 is known to play a prominent part in the homology of $\mathrm{M}_{n}$. Specifically, write $v_{n}=\left\lfloor\frac{n-2}{3}\right\rfloor=\left\lceil\frac{n-4}{3}\right\rceil$. By a result due to Björner, Lovász, Vrećica, and Živaljević [4], the reduced homology group $\tilde{H}_{i}\left(\mathrm{M}_{n} ; \mathbb{Z}\right)$ is zero whenever $i<v_{n}$. Bouc [5] showed that $\tilde{H}_{v_{n}}\left(\mathrm{M}_{n} ; \mathbb{Z}\right) \cong \mathbb{Z}_{3}$ whenever $n=3 k+1 \geq 7$ and that $\tilde{H}_{v_{n}}\left(\mathrm{M}_{n} ; \mathbb{Z}\right)$ has exponent dividing nine whenever $n=3 k \geq 12$. Shareshian and Wachs extended and improved Bouc's result:

Theorem 1.1 (Shareshian and Wachs $[20]) \tilde{H}_{v_{n}}\left(\mathrm{M}_{n} ; \mathbb{Z}\right)$ is an elementary 3-group for $n \in\{7,10,12,13\}$ and also for $n \geq 15$. The torsion subgroup of $\tilde{H}_{v_{n}}\left(\mathrm{M}_{n} ; \mathbb{Z}\right)$ is again an elementary 3-group for $n \in\{9,11\}$ and zero for $n \in\{1,2,3,4,5,6,8\}$. For the remaining case $n=14, \tilde{H}_{v_{n}}\left(\mathrm{M}_{n} ; \mathbb{Z}\right)$ is a finite group with nonvanishing 3-torsion.

To prove that the group $\tilde{H}_{v_{n}}\left(\mathrm{M}_{n} ; \mathbb{Z}\right)$ is elementary for $n \equiv 0(\bmod 3)$ and $n \geq 12$ and for $n \equiv 2(\bmod 3)$ and $n \geq 17$, Shareshian and Wachs relied on a computer calculation of the group $\tilde{H}_{3}\left(\mathrm{M}_{12} ; \mathbb{Z}\right)$. The existence of 3 -torsion in the homology of $\mathrm{M}_{9}$ and $\mathrm{M}_{11}$ also relied on such calculations. Unfortunately, attempts to stretch this computer approach beyond $n=12$ have failed; the size of $\mathrm{M}_{n}$ is too large for the existing software to handle when $n \geq 13$. In particular, the structure of the bottom nonvanishing homology group of $\mathrm{M}_{14}$ has remained a mystery.

For completeness, let us mention that there is 3-torsion also in higher-degree homology groups [14]. More precisely, there is 3-torsion in $\tilde{H}_{d}\left(\mathrm{M}_{n} ; \mathbb{Z}\right)$ whenever $v_{n} \leq d \leq \frac{n-6}{2}$. As a consequence, since there is homology in degree $\left\lfloor\frac{n-3}{2}\right\rfloor$ but not above this degree [5], there is 3-torsion in almost all nonvanishing homology groups of $\mathrm{M}_{n}$, the only exceptions being the top degree $\left\lfloor\frac{n-3}{2}\right\rfloor$ and possibly the degree $\frac{n-5}{2}$ just below it for odd $n$. The homology in the latter degree is known to contain 3 -torsion for $n \in\{7,9,11,13\}$; see Proposition 3.8 for the case $n=13$. A complete description of the homology groups of $\mathrm{M}_{n}$ is known only for $n \leq 12$; see Table 1 .

The appearance of 3-torsion being so prominent, it makes sense to ask whether this is the only kind of torsion that appears in $\mathrm{M}_{n}$ for any given $n$. Indeed, Babson, Björner, Linusson, Shareshian, and Welker [3] asked this very question. Based on the overwhelming evidence presented in Theorem 1.1, Shareshian and Wachs [20] conjectured that $\tilde{H}_{v_{14}}\left(\mathrm{M}_{14} ; \mathbb{Z}\right)=\tilde{H}_{4}\left(\mathrm{M}_{14} ; \mathbb{Z}\right)$ is an elementary 3-group. Surprisingly, the conjecture turns out to be false:

Theorem $1.2 \tilde{H}_{4}\left(\mathrm{M}_{14} ; \mathbb{Z}\right)$ is a finite group of exponent a multiple of 15 .

Theorem 1.2 being just one specific example, the question of Babson et al. remains unanswered in general; we do not know whether there is other torsion than 3-torsion in the homology of other matching complexes. See Section 4 for some discussion.

To prove Theorem 1.2, we use a result due to Andersen about the homology of $\mathrm{BD}_{7}^{2}$ : 
Table 1 The homology of $\mathrm{M}_{n}$ for $n \leq 14$; see Wachs [21] for explanation of the parts that are not explained in the present note. $T_{1}$ and $T_{2}$ are nontrivial finite groups of exponent a multiple of 3 and 15 , respectively; see Proposition 3.8 and Theorem 1.2

\begin{tabular}{rllllll}
\hline$\tilde{H}_{i}\left(\mathrm{M}_{n} ; \mathbb{Z}\right)$ & $i=0$ & 1 & 2 & 3 & 4 & 5 \\
\hline$n=3$ & $\mathbb{Z}^{2}$ & - & - & - & - & - \\
4 & $\mathbb{Z}^{2}$ & - & - & - & - & - \\
5 & - & $\mathbb{Z}^{6}$ & - & - & - & - \\
6 & - & $\mathbb{Z}^{16}$ & - & - & - & - \\
7 & - & $\mathbb{Z}_{3}$ & $\mathbb{Z}^{20}$ & - & - & - \\
8 & - & - & $\mathbb{Z}^{132}$ & - & - & - \\
9 & - & - & $\mathbb{Z}_{3}^{8} \oplus \mathbb{Z}^{42}$ & $\mathbb{Z}^{70}$ & - & - \\
10 & - & - & $\mathbb{Z}_{3}$ & $\mathbb{Z}^{1216}$ & - & - \\
11 & - & - & - & $\mathbb{Z}_{3}^{45} \oplus \mathbb{Z}^{1188}$ & $\mathbb{Z}^{252}$ & - \\
12 & - & - & - & $\mathbb{Z}_{3}^{56}$ & $\mathbb{Z}^{12440}$ & - \\
13 & - & - & - & $\mathbb{Z}_{3}$ & $T_{1} \oplus \mathbb{Z}^{24596}$ & $\mathbb{Z}^{924}$ \\
14 & - & - & - & - & $T_{2}$ & $\mathbb{Z}^{138048}$ \\
\hline
\end{tabular}

Theorem 1.3 (Andersen [1]) We have that

$$
\tilde{H}_{i}\left(\mathrm{BD}_{7}^{2} ; \mathbb{Z}\right) \cong \begin{cases}\mathbb{Z}_{5} & \text { if } i=4 \\ \mathbb{Z}^{732} & \text { if } i=5 \\ 0 & \text { otherwise. }\end{cases}
$$

Remark We have verified Theorem 1.3 using the Homology computer program [11].

Let $\mathfrak{S}_{n}$ be the symmetric group on $n$ elements. We relate Andersen's result to the homology of $\mathrm{M}_{14}$ via a map $\pi^{*}$ from $\tilde{H}_{4}\left(\mathrm{M}_{14} ; \mathbb{Z}\right)$ to $\tilde{H}_{4}\left(\mathrm{BD}_{7}^{2} ; \mathbb{Z}\right)$; this map is induced by the natural action on $\mathrm{M}_{14}$ by the Young group $\left(\mathfrak{S}_{2}\right)^{7}$. Using a standard representation-theoretic argument, we construct an "inverse" $\varphi^{*}$ of $\pi^{*}$ with the property that $\pi^{*} \circ \varphi^{*}(z)=\left|\left(\mathfrak{S}_{2}\right)^{7}\right| \cdot z$ for all $z \in \tilde{H}_{4}\left(\mathrm{BD}_{7}^{2} ; \mathbb{Z}\right)$. To conclude the proof, one observes that $\varphi^{*}(z)$ is nonzero unless the order of $z$ divides the order of $\left(\mathfrak{S}_{2}\right)^{7}$. Since the latter order is 128 , the image under $\varphi^{*}$ of any nonzero element of order five is again a nonzero element of order five.

Using computer, we have also been able to deduce that there is 3-torsion in the homology of $\tilde{H}_{4}\left(\mathrm{BD}_{7}^{\left(2^{3} 1^{7}\right)} ; \mathbb{Z}\right)$. where $\left(2^{3} 1^{7}\right)$ denotes the sequence $(2,2,2,1,1,1,1,1$, $1,1)$. An argument similar to the one above yields that $\tilde{H}_{4}\left(\mathrm{M}_{13} ; \mathbb{Z}\right)$ contains 3 -torsion. By the results of Bouc [5], we already know that $\tilde{H}_{3}\left(\mathrm{M}_{13} ; \mathbb{Z}\right) \cong \mathbb{Z}_{3}$.

For the sake of generality, we describe our simple representation-theoretic construction in terms of an arbitrary finite group acting on a chain complex of abelian groups; see Section 2. The particular case that we are interested in is discussed in Section 3. In Section 4, we make some remarks and discuss potential improvements and generalizations of our result. 


\section{Group actions on chain complexes}

We recall some elementary properties of group actions on chain complexes; see Bredon [6] for a more thorough treatment. Let

$$
\mathcal{C}: \cdots \stackrel{\partial_{d+1}}{\longrightarrow} C_{d} \stackrel{\partial_{d}}{\longrightarrow} C_{d-1} \stackrel{\partial_{d-1}}{\longrightarrow} C_{d-2} \stackrel{\partial_{d-2}}{\longrightarrow} \cdots
$$

be a chain complex of abelian groups. Let $G$ be a group acting on $\mathcal{C}$, meaning the following for each $k \in \mathbb{Z}$ :

- Every $g \in G$ defines a degree-preserving automorphism on $\mathcal{C}$.

- For every $g, h \in G$ and $c \in C_{k}$, we have that $g(h(c))=(g h)(c)$.

- For every $g \in G$ and $c \in C_{k}$, we have that $\partial_{k}(g(c))=g\left(\partial_{k}(c)\right)$.

Let $C_{d}^{G}$ be the subgroup of $C_{d}$ generated by $\left\{c-g(c): c \in C_{d}, g \in G\right\}$ and let $\mathcal{C}^{G}$ be the corresponding chain complex. $\mathcal{C}^{G}$ is indeed a chain complex, because $\partial_{d}(c-g(c))=\partial_{d}(c)-g\left(\partial_{d}(c)\right) \in C_{d-1}^{G}$ whenever $c \in C_{d}$. Writing $C_{d} / G=C_{d} / C_{d}^{G}$, we obtain the quotient chain complex

$$
\mathcal{C} / G: \cdots \stackrel{\partial_{d+1}}{\longrightarrow} C_{d} / G \stackrel{\partial_{d}}{\longrightarrow} C_{d-1} / G \stackrel{\partial_{d-1}}{\longrightarrow} C_{d-2} / G \stackrel{\partial_{d-2}}{\longrightarrow} \cdots
$$

In particular, we have the following exact sequence of homology groups for each $d$ :

$$
H_{d+1}(\mathcal{C} / G) \longrightarrow H_{d}\left(\mathcal{C}^{G}\right) \longrightarrow H_{d}(\mathcal{C}) \stackrel{\pi_{d}^{*}}{\longrightarrow} H_{d}(\mathcal{C} / G) \longrightarrow H_{d-1}\left(\mathcal{C}^{G}\right)
$$

$\pi_{d}^{*}$ is the map induced by the natural projection map $\pi_{d}: C_{d} \rightarrow C_{d} / G$.

From now on, assume that $G$ is finite. For an element $c \in C_{d}$, let $[c]$ denote the corresponding element in $C_{d} / G ;[c]=c+C_{d}^{G}$. Define $\mathcal{O}_{G}(c)=\sum_{g \in G} g(c)$. Clearly, $\mathcal{O}_{G}(c)=0$ for all $c \in C_{d}^{G}$ and $\mathcal{O}_{G}$ commutes with $\partial_{d}$. Let $\varphi_{d}: C_{d} / G \rightarrow C_{d}$ be the homomorphism defined by $\varphi_{d}([c])=\mathcal{O}_{G}(c)$. Since $\mathcal{O}_{G}$ vanishes on $C_{d}^{G}$, we have that $\varphi_{d}$ is well-defined. Moreover, $\partial_{d} \circ \varphi_{d}=\varphi_{d-1} \circ \partial_{d}$, because

$$
\partial_{d}\left(\varphi_{d}([c])\right)=\partial_{d}\left(\mathcal{O}_{G}(c)\right)=\mathcal{O}_{G}\left(\partial_{d}(c)\right)=\varphi_{d-1}\left(\left[\partial_{d}(c)\right]\right)=\varphi_{d-1}\left(\partial_{d}([c])\right) .
$$

Let $\varphi_{d}^{*}: H_{d}(\mathcal{C} / G) \rightarrow H_{d}(\mathcal{C})$ be the map induced by $\varphi_{d}$; this is a well-defined homomorphism by the above discussion.

Lemma 2.1 The kernel of $\varphi_{d}^{*}$ has finite exponent dividing $|G|$. As a consequence, if the torsion subgroup of $H_{d}(\mathcal{C})$ has finite exponent $e(e=1$ if there is no torsion), then the exponent of the torsion subgroup of $H_{d}(\mathcal{C} / G)$ is also finite and divides $|G| \cdot e$.

Proof Let $c \in H_{d}(\mathcal{C})$. Since $\left[\varphi_{d}^{*}([c])\right]=\left[\mathcal{O}_{G}(c)\right]=|G| \cdot[c]$ and $0=\left[e \cdot \varphi_{d}^{*}([c])\right]=$ $e \cdot|G| \cdot[c]$, we are done. 


\section{Detecting 5-torsion in the homology of $\mathrm{M}_{14}$}

Let $\lambda=\left(\lambda_{1}, \ldots, \lambda_{n}\right)$ be a sequence of nonnegative integers summing to $N$. Define $\mathfrak{S}_{\lambda}$ to be the Young group $\mathfrak{S}_{\lambda_{1}} \times \cdots \times \mathfrak{S}_{\lambda_{n}}$. Write $[N]$ as a disjoint union $\bigcup_{i=1}^{n} U_{i}$ such that $\left|U_{i}\right|=\lambda_{i}$ for each $i$ and let $\mathfrak{S}_{\lambda_{i}}$ act on $U_{i}$ in the natural manner for each $i$. This yields an action of $\mathfrak{S}_{\lambda}$ on $[N]$, and this action induces an action on the chain complex $\tilde{\mathcal{C}}\left(\mathrm{M}_{N}\right)$. In particular, we have the following result:

Lemma 3.1 Let $\varphi_{d}^{*}: H_{d}\left(\tilde{\mathcal{C}}\left(\mathrm{M}_{N}\right) / \mathfrak{S}_{\lambda}\right) \rightarrow \tilde{H}_{d}\left(\mathrm{M}_{N}\right)$ be defined as in Lemma 2.1. Then the kernel of $\varphi_{d}^{*}$ has finite exponent dividing $\prod_{i=1}^{n} \lambda !$.

Proof This is an immediate consequence of Lemma 2.1.

Let $\Delta_{\lambda}$ be the subfamily of $\mathrm{M}_{N}$ consisting of all $\sigma$ such that there are two distinct edges $a b$ and $c d$ in $\sigma$ with the property that $\{a, c\} \subseteq U_{i}$ and $\{b, d\} \subseteq U_{j}$ for some $i$ and $j$ (possibly equal). Write $\Gamma_{\lambda}=\mathrm{M}_{N} \backslash \Delta_{\lambda}$; this is a simplicial complex. Define $\kappa:[N] \rightarrow[n]$ by $\kappa^{-1}(\{i\})=U_{i}$. Extend $\kappa$ to $\Gamma_{\lambda}$ by defining

$$
\kappa\left(\left\{a_{1} b_{1}, \ldots, a_{r} b_{r}\right\}\right)=\left\{\kappa\left(a_{1}\right) \kappa\left(b_{1}\right), \ldots, \kappa\left(a_{r}\right) \kappa\left(b_{r}\right)\right\} .
$$

Lemma 3.2 We have that $\kappa$ is a dimension-preserving surjective map from $\Gamma_{\lambda}$ to $\mathrm{BD}_{n}^{\lambda}$.

Proof To see that $\kappa$ is dimension-preserving, note that $|\kappa(\sigma)|=|\sigma|$ whenever $\sigma$ belongs to $\Gamma_{\lambda}$. Namely, there are no multiple edges or multiple loops in $\kappa(\sigma)$ by definition of $\Gamma_{\lambda}$. Moreover, $\kappa(\sigma)$ belongs to $\mathrm{BD}_{n}^{\lambda}$, because for each $i \in[n]$, the degree in $\kappa(\sigma)$ of the vertex $i$ equals the sum of the degrees in $\sigma$ of all vertices in $U_{i}$; this is at most $\left|U_{i}\right|=\lambda_{i}$. To prove surjectivity, use a simple induction argument over $\lambda$; remove one edge at a time from a given graph in $\mathrm{BD}_{n}^{\lambda}$.

Lemma 3.3 For $\sigma, \tau \in \Gamma_{\lambda}$, we have that $\kappa(\sigma)=\kappa(\tau)$ if and only if there is a $g$ in $\mathfrak{S}_{\lambda}$ such that $g(\sigma)=\tau$.

Proof Clearly, $\kappa(\sigma)=\kappa(g(\sigma))$ for all $\sigma \in \Gamma_{\lambda}$ and $g \in \mathfrak{S}_{\lambda}$. For the other direction, write $\sigma=\left\{a_{j} b_{j}: j \in J\right\}$ and $\tau=\left\{a_{j}^{\prime} b_{j}^{\prime}: j \in J\right\}$, where $\kappa\left(a_{j}\right)=\kappa\left(a_{j}^{\prime}\right)$ and $\kappa\left(b_{j}\right)=$ $\kappa\left(b_{j}^{\prime}\right)$ for each $j \in J$. Define $g\left(a_{j}\right)=a_{j}^{\prime}$ and $g\left(b_{j}\right)=b_{j}^{\prime}$ for each $j \in J$ and extend $g$ to a permutation on $[N]$ such that $g\left(U_{i}\right)=U_{i}$ for each $i \in[n]$. One easily checks that $g$ has the desired properties.

For a set $\sigma$, we let $\sigma^{\text {or }}$ denote the oriented simplex corresponding to $\sigma$; fixing an order of the elements in $\sigma$, this is well-defined. Given an oriented simplex $\sigma^{o r}=$ $a_{1} b_{1} \wedge \cdots \wedge a_{r} b_{r}$, we define

$$
\kappa\left(\sigma^{o r}\right)=\kappa\left(a_{1}\right) \kappa\left(b_{1}\right) \wedge \cdots \wedge \kappa\left(a_{r}\right) \kappa\left(b_{r}\right),
$$

thereby preserving orientation. Extend $\kappa$ linearly to a homomorphism $\tilde{\mathcal{C}}\left(\Gamma_{\lambda}\right) \rightarrow$ $\tilde{\mathcal{C}}\left(\mathrm{BD}_{n}^{\lambda}\right)$. 
Lemma 3.4 The map $\hat{\kappa}: \tilde{\mathcal{C}}\left(\Gamma_{\lambda}\right) / \mathfrak{S}_{\lambda} \rightarrow \tilde{\mathcal{C}}\left(\mathrm{BD}_{n}^{\lambda}\right)$ defined as $\hat{\kappa}([c])=\kappa(c)$ is a chain complex isomorphism.

Proof First of all, one easily checks that $\hat{\kappa}$ is well-defined and commutes with the boundary operator; for the former property, note that $\kappa\left(\sigma^{o r}\right)=\kappa\left(g\left(\sigma^{o r}\right)\right)$ for all $g \in \mathfrak{S}_{\lambda}$ and $\sigma \in \Gamma_{\lambda}$. Moreover, $\hat{\kappa}$ is surjective, because $\kappa$ is surjective by Lemma 3.2. Finally, to see that $\hat{\kappa}$ is injective, define $\mu: \tilde{\mathcal{C}}\left(\operatorname{BD}_{n}^{\lambda}\right) \rightarrow \tilde{\mathcal{C}}\left(\Gamma_{\lambda}\right) / \mathfrak{S}_{\lambda}$ as $\mu\left(c^{\prime}\right)=[c]$, where $c$ is any element in $\tilde{\mathcal{C}}\left(\Gamma_{\lambda}\right)$ such that $\kappa(c)=c^{\prime}$; this is well-defined by Lemma 3.3. Since $\mu \circ \hat{\kappa}([c])=\mu(\kappa(c))=[c]$, injectivity follows.

Theorem 3.5 We have the chain complex isomorphism

$$
\tilde{\mathcal{C}}\left(\mathrm{M}_{N}\right) / \mathfrak{S}_{\lambda} \cong \tilde{\mathcal{C}}\left(\mathrm{BD}_{n}^{\lambda}\right) \oplus \tilde{\mathcal{C}}\left(\Delta_{\lambda}\right) / \mathfrak{S}_{\lambda}
$$

Proof By Lemma 3.4, it suffices to prove that

$$
\tilde{\mathcal{C}}\left(\mathrm{M}_{N}\right) / \mathfrak{S}_{\lambda} \cong \tilde{\mathcal{C}}\left(\Gamma_{\lambda}\right) / \mathfrak{S}_{\lambda} \oplus \tilde{\mathcal{C}}\left(\Delta_{\lambda}\right) / \mathfrak{S}_{\lambda}
$$

Clearly, the boundary in $\tilde{\mathcal{C}}\left(\mathrm{M}_{N}\right) / \mathfrak{S}_{\lambda}$ of any element in $\tilde{\mathcal{C}}\left(\Gamma_{\lambda}\right) / \mathfrak{S}_{\lambda}$ is again an element in $\tilde{\mathcal{C}}\left(\Gamma_{\lambda}\right) / \mathfrak{S}_{\lambda}, \Gamma_{\lambda}$ being a subcomplex of $\mathrm{M}_{N}$. It remains to prove that $\left[\partial\left(\sigma^{o r}\right)\right] \in$ $\tilde{\mathcal{C}}\left(\Delta_{\lambda}\right) / \mathfrak{S}_{\lambda}$ for each $\sigma \in \Delta_{\lambda}$. Write $\sigma^{\text {or }}=a_{1} b_{1} \wedge a_{2} b_{2} \wedge \tau^{o r}$, where $a_{1}, a_{2} \in U_{i}$ and $b_{1}, b_{2} \in U_{j}$ for some $i$ and $j$. We obtain that

$$
\partial\left(\sigma^{o r}\right)=a_{2} b_{2} \wedge \tau^{o r}-a_{1} b_{1} \wedge \tau^{o r}+a_{1} b_{1} \wedge a_{2} b_{2} \wedge \partial\left(\tau^{o r}\right) .
$$

Since the group element $\left(a_{1}, a_{2}\right)\left(b_{1}, b_{2}\right)$ belongs to $\mathfrak{S}_{\lambda}$ and transforms $a_{1} b_{1} \wedge \tau^{o r}$ into $a_{2} b_{2} \wedge \tau^{o r}$, it follows that

$$
\partial\left(\left[\sigma^{o r}\right]\right)=\left[a_{1} b_{1} \wedge a_{2} b_{2} \wedge \partial\left(\tau^{o r}\right)\right]
$$

which is indeed an element in $\tilde{\mathcal{C}}\left(\Delta_{\lambda}\right) / \mathfrak{S}_{\lambda}$.

Remark. One may note that $\tilde{\mathcal{C}}\left(\Delta_{\lambda}\right) / \mathfrak{S}_{\lambda}$ is a chain complex of elementary 2-groups. Namely, with notation as in the above proof, we have that $\left(a_{1}, a_{2}\right)\left(b_{1}, b_{2}\right)$ maps $\sigma^{o r}=$ $a_{1} b_{1} \wedge a_{2} b_{2} \wedge \tau^{o r}$ to $a_{2} b_{2} \wedge a_{1} b_{1} \wedge \tau^{o r}=-\sigma^{o r}$. As a consequence, $\left[\sigma^{o r}\right]=-\left[\sigma^{o r}\right]$, which implies that $2\left[\sigma^{o r}\right]=0$.

Theorem 3.6 There is a homomorphism $\tilde{H}_{d}\left(\mathrm{BD}_{n}^{\lambda}\right) \rightarrow \tilde{H}_{d}\left(\mathrm{M}_{N}\right)$ such that the kernel has finite exponent dividing $\prod_{i=1}^{n} \lambda$ !.

Proof This follows immediately from Lemma 3.1 and Theorem 3.5.

Let us summarize the situation. 
Corollary 3.7 We have a long exact sequence

$$
\begin{aligned}
& \cdots \quad \longrightarrow H_{d+1}\left(\tilde{\mathcal{C}}\left(\mathrm{M}_{N}\right) / \mathfrak{S}_{\lambda}\right) \\
& \longrightarrow H_{d}\left(\tilde{\mathcal{C}}\left(\mathrm{M}_{N}\right)^{\mathfrak{S}_{\lambda}}\right) \longrightarrow \tilde{H}_{d}\left(\mathrm{M}_{N}\right) \stackrel{\pi_{d}^{*}}{\longrightarrow} H_{d}\left(\tilde{\mathcal{C}}\left(\mathrm{M}_{N}\right) / \mathfrak{S}_{\lambda}\right) \\
& \longrightarrow H_{d-1}\left(\tilde{\mathcal{C}}_{d}\left(\mathrm{M}_{N}\right)^{\mathfrak{S}_{\lambda}}\right) \longrightarrow \cdots
\end{aligned}
$$

where

$$
H_{d}\left(\tilde{\mathcal{C}}\left(\mathrm{M}_{N}\right) / \mathfrak{S}_{\lambda}\right) \cong \tilde{H}_{d}\left(\mathrm{BD}_{n}^{\lambda}\right) \oplus H_{d}\left(\tilde{\mathcal{C}}\left(\Delta_{\lambda}\right) / \mathfrak{S}_{\lambda}\right)
$$

and $\pi_{d}^{*}$ has an "inverse" $\varphi_{d}^{*}$ satisfying $\pi_{d}^{*} \circ \varphi_{d}^{*}=\prod_{i=1}^{n} \lambda$ ! $\cdot \mathrm{id}$. In particular, if $\prod_{i=1}^{n} \lambda$ ! is a unit in the underlying coefficient ring, then

$$
\tilde{H}_{d}\left(\mathrm{M}_{N}\right) \cong \tilde{H}_{d}\left(\mathrm{BD}_{n}^{\lambda}\right) \oplus H_{d}\left(\tilde{\mathcal{C}}\left(\mathrm{M}_{N}\right)^{\mathfrak{S}_{\lambda}}\right)
$$

For the final statement, note that $\tilde{\mathcal{C}}\left(\Delta_{\lambda}\right) / \mathfrak{S}_{\lambda}$ is zero if 2 is a unit in the underlying coefficient ring or if $\lambda=(1, \ldots, 1)$.

Proof of Theorem 1.2 By Theorem 1.1, we already know that there are elements of order three in $\tilde{H}_{4}\left(\mathrm{M}_{14} ; \mathbb{Z}\right)$ and that the group is finite. Applying Theorem 1.3, we obtain that the exponent of $\tilde{H}_{4}\left(\mathrm{BD}_{7}^{2} ; \mathbb{Z}\right)$ is five. Selecting $\lambda=(2,2,2,2,2,2,2)$ and noting that $\prod_{i} \lambda_{i} !=128$ and $\operatorname{gcd}(5,128)=1$, we are done by Theorem 3.6.

Let $\left(2^{a} 1^{b}\right)$ denote the sequence consisting of $a$ occurrences of the value 2 and $b$ occurrences of the value 1 . One may try to obtain further information about the homology of $\mathrm{M}_{14}$ by computing the homology of $\mathrm{BD}_{14-a}^{\left(2^{a} 1^{14-2 a}\right)}$ for $a \leq 6$. The ideal, of course, would be to compute the homology of $\mathrm{M}_{14}$ directly, but this appears to be beyond the capacity of today's (standard) computers. Using the computer program CHomP [18], we managed to compute the $\mathbb{Z}_{p}$-homology of $\mathrm{BD}_{8}^{\left(2^{6} 1^{2}\right)}$ for $p \in\{2,3,5\}$, and the results suggest that $\tilde{H}_{4}\left(\mathrm{BD}_{8}^{\left(2^{6} 1^{2}\right)} ; \mathbb{Z}\right) \cong \tilde{H}_{4}\left(\mathrm{BD}_{7}^{2} ; \mathbb{Z}\right) \cong \mathbb{Z}$. In particular, it seems that we cannot gather any additional information about the homology of $\mathrm{M}_{14}$ from that of $\mathrm{BD}_{8}^{\left(2^{6} 1^{2}\right)}$.

Via a calculation with the Homology computer program [11], we discovered that

$$
\tilde{H}_{4}\left(\mathrm{BD}_{11}^{\left(2^{2} 1^{9}\right)} ; \mathbb{Z}\right) \cong \mathbb{Z}_{3}^{10} \oplus \mathbb{Z}^{6142}
$$

By Theorem 3.6 and well-known properties of the rational homology of $\mathrm{M}_{13}$ [5], this yields the following result:

Proposition 3.8 We have that $\tilde{H}_{4}\left(\mathrm{M}_{13} ; \mathbb{Z}\right) \cong T \oplus \mathbb{Z}^{24596}$, where $T$ is a finite group containing $\mathbb{Z}_{3}^{10}$ as a subgroup.

See Tables 2 and 3 for more information about torsion in the homology of $\mathrm{BD}_{a+b}^{2^{a} 1^{b}}$ for small values of $a$ and $b$. The numerical data in Table 2 suggests that the Sylow 3 -subgroup of $\tilde{H}_{(n-5) / 2}\left(\mathrm{BD}_{n-a}^{2^{a} 1^{n-2 a}} ; \mathbb{Z}\right)$ is an elementary 3-group of rank $\left(\begin{array}{c}n-a-1 \\ (n+5) / 2\end{array}\right)$. 
Table 2 Torsion subgroup of $\tilde{H}_{i}\left(\mathrm{BD}_{n-a}^{2^{a} 1^{n-2 a}} ; \mathbb{Z}\right)$ for $n=2 i+5$

\begin{tabular}{rllllllll}
\hline & $a=0$ & 1 & 2 & 3 & 4 & 5 & 6 & 7 \\
\hline$n=3$ & 0 & 0 & - & - & - & - & - & - \\
5 & 0 & 0 & 0 & - & - & - & - & - \\
7 & $\mathbb{Z}_{3}$ & 0 & 0 & 0 & - & - & - & - \\
9 & $\mathbb{Z}_{3}^{8}$ & $\mathbb{Z}_{3}$ & 0 & 0 & 0 & - & - & - \\
11 & $\mathbb{Z}_{3}^{45}$ & $\mathbb{Z}_{3}^{9}$ & $\mathbb{Z}_{3}$ & 0 & 0 & 0 & - & - \\
13 & $?$ & $?$ & $\mathbb{Z}_{3}^{10}$ & $\mathbb{Z}_{3}$ & 0 & 0 & 0 & - \\
15 & $?$ & $?$ & $?$ & $?$ & $?$ & $?$ & 0 & 0 \\
\hline
\end{tabular}

Table 3 Torsion subgroup of $\tilde{H}_{i}\left(\mathrm{BD}_{n-a}^{2^{a} 1^{n-2 a}} ; \mathbb{Z}\right)$ for $n=2 i+6$

\begin{tabular}{rllllllll}
\hline & $a=0$ & 1 & 2 & 3 & 4 & 5 & 6 & 7 \\
\hline$n=2$ & 0 & 0 & - & - & - & - & - & - \\
4 & 0 & 0 & 0 & - & - & - & - & - \\
6 & 0 & 0 & 0 & 0 & - & - & - & - \\
8 & 0 & 0 & 0 & 0 & 0 & - & - & - \\
10 & $\mathbb{Z}_{3}$ & 0 & 0 & 0 & 0 & 0 & - & - \\
12 & $\mathbb{Z}_{3}^{56}$ & $\mathbb{Z}_{3}^{10}$ & $\mathbb{Z}_{3}$ & 0 & 0 & 0 & 0 & - \\
14 & $?$ & $?$ & $?$ & $?$ & $?$ & $?$ & $\mathbb{Z}_{5} ?$ & $\mathbb{Z}_{5}$ \\
\hline
\end{tabular}

\section{Remarks and further directions}

Using CHomP [18], we managed to compute a generator $\gamma^{\prime}$ for the homology group $\tilde{H}_{4}\left(\mathrm{BD}_{7}^{2} ; \mathbb{Z}\right) \cong \mathbb{Z}_{5}$;

$$
\begin{aligned}
\gamma^{\prime}= & ([12,45,23]+[12,23,34]+[12,34,15]+[12,15,33]+[12,33,45] \\
& +[22,33,15]+[22,15,34]+[22,34,11]+[22,11,45]+[22,45,33] \\
& +[11,23,45]+[11,34,23]) \wedge(46-66) \wedge(57-77) ;
\end{aligned}
$$

$[a b, c d, e f]=a b \wedge c d \wedge e f$. Note that $\gamma^{\prime}=\gamma / \mathfrak{S}_{\left(2^{7}\right)}$, where

$$
\begin{aligned}
\gamma= & ([1 \hat{2}, 5 \hat{4}, 2 \hat{3}]+[1 \hat{2}, 2 \hat{3}, 3 \hat{4}]+[1 \hat{2}, 3 \hat{4}, 5 \hat{1}]+[1 \hat{2}, 5 \hat{1}, 3 \hat{3}]+[1 \hat{2}, 3 \hat{3}, 5 \hat{4}] \\
& +[2 \hat{2}, 3 \hat{3}, 5 \hat{1}]+[2 \hat{2}, 5 \hat{1}, 3 \hat{4}]+[2 \hat{2}, 3 \hat{4}, 1 \hat{1}]+[2 \hat{2}, 1 \hat{1}, 5 \hat{4}]+[2 \hat{2}, 5 \hat{4}, 3 \hat{3}] \\
& +[1 \hat{1}, 2 \hat{3}, 5 \hat{4}]+[1 \hat{1}, 3 \hat{4}, 2 \hat{3}]) \wedge(4 \hat{6}-6 \hat{6}) \wedge(7 \hat{5}-7 \hat{7}) .
\end{aligned}
$$

Here, $\hat{i}$ denotes the vertex $i+7$, and the group action is given by the partition $\left\{U_{i}\right.$ : $i \in[7]\}$, where $U_{i}=\{i, \hat{i}\}$. Since $\tilde{H}_{4}\left(\mathrm{M}_{14} ; \mathbb{Z}\right)$ is finite, we conclude that $\gamma$ has finite exponent a multiple of five in $\tilde{H}_{4}\left(\mathrm{M}_{14} ; \mathbb{Z}\right)$. Note that we may view $\gamma$ as the product of one cycle in $\tilde{H}_{2}\left(\mathrm{M}_{8} ; \mathbb{Z}\right)$ and two cycles in $\tilde{H}_{0}\left(\mathrm{M}_{3} ; \mathbb{Z}\right)$ (defined on three disjoint vertex sets).

Another observation is that we have the following portion of the long exact sequence for the pair $\left(\mathrm{M}_{14}, \mathrm{M}_{13}\right)$ :

$$
\tilde{H}_{4}\left(\mathrm{M}_{13} ; \mathbb{Z}\right) \longrightarrow \tilde{H}_{4}\left(\mathrm{M}_{14} ; \mathbb{Z}\right) \longrightarrow \bigoplus_{13} \tilde{H}_{3}\left(\mathrm{M}_{12} ; \mathbb{Z}\right) ;
$$


see Bouc [5]. Since $\tilde{H}_{3}\left(\mathrm{M}_{12} ; \mathbb{Z}\right)$ is an elementary 3 -group by the data in Table 1 , this yields that there must be some element $\delta$ in $\tilde{H}_{4}\left(\mathrm{M}_{13} ; \mathbb{Z}\right)$ such that $\delta$ is identical in $\tilde{H}_{4}\left(\mathrm{M}_{14} ; \mathbb{Z}\right)$ to $\gamma$ or $3 \gamma$. Obviously, the exponent of $\delta$ in $\tilde{H}_{4}\left(\mathrm{M}_{13} ; \mathbb{Z}\right)$ is either infinite or a nonzero multiple of five; we conjecture the former.

As mentioned in Section 1, we do not know whether there is 5-torsion in the homology of $\mathrm{M}_{n}$ when $n \geq 13$ and $n \neq 14$. We would indeed have such torsion for all even $n \geq 16$ if

$$
\tilde{H}_{d}\left(\mathrm{M}_{n} ; \mathbb{Z}\right) \cong \tilde{H}_{d}\left(\mathrm{M}_{n} \backslash e ; \mathbb{Z}\right) \oplus \tilde{H}_{d-1}\left(\mathrm{M}_{n-2} ; \mathbb{Z}\right)
$$

for all even $n \geq 16$ and $d=n / 2-3$. Here, $e$ is the edge between $n-1$ and $n$ and $\mathrm{M}_{n} \backslash e$ is the complex obtained from $\mathrm{M}_{n}$ by removing the 0 -cell $e$. Using computer, we have verified (1) for all $(n, d)$ such that $n \leq 11$ and $d \geq 0$. Note that (1) would follow if the sequence

$$
0 \longrightarrow \tilde{H}_{d}\left(\mathrm{M}_{n} \backslash e ; \mathbb{Z}\right) \longrightarrow \tilde{H}_{d}\left(\mathrm{M}_{n} ; \mathbb{Z}\right) \longrightarrow \tilde{H}_{d-1}\left(\mathrm{M}_{n-2} ; \mathbb{Z}\right) \longrightarrow 0
$$

turned out to be split exact. By the long exact sequence for the pair $\left(\mathrm{M}_{n}, \mathrm{M}_{n} \backslash e\right)$, the mid-portion of this sequence is indeed exact.

\section{References}

1. Andersen, J.L.: Determinantal rings associated with symmetric matrices: a counterexample. Ph.D. Thesis, University of Minnesota (1992)

2. Athanasiadis, C.A.: Decompositions and connectivity of matching and chessboard complexes. Discrete Comput. Geom. 31(3), 395-403 (2004)

3. Babson, E., Björner, A., Linusson, S., Shareshian, J., Welker, V.: Complexes of not $i$-connected graphs. Topology 38(2), 271-299 (1999)

4. Björner, A., Lovász, L., Vrećica, S.T., Živaljević, R.T.: Chessboard complexes and matching complexes. J. London Math. Soc. 49(2), 25-39 (1994)

5. Bouc, S.: Homologie de certains ensembles de 2-sous-groupes des groupes symétriques. J. Algebra 150, 187-205 (1992)

6. Bredon, G.E.: Introduction to Compact Transformation Groups. Academic Press, San Diego (1972)

7. Dong, X.: The topology of bounded degree graph complexes and finite free resolutions. Ph.D. Thesis, University of Minnesota (2001)

8. Dong, X., Wachs, M.L.: Combinatorial Laplacian of the matching complex. Electronic J. Combin. 9(1), R17 (2002)

9. Friedman, J., Hanlon, P.: On the Betti numbers of chessboard complexes. J. Algebraic Combin. 8, 193-203 (1998)

10. Garst, P.F.: Cohen-Macaulay complexes and group actions. Ph.D. Thesis, University of WisconsinMadison (1979)

11. Dumas, J.-G., Heckenbach, F., Saunders, B.D., Welker, V.: Simplicial Homology, a share package for GAP (2000)

12. Jonsson, J.: Simplicial complexes of graphs. Doctoral Thesis, KTH (2005)

13. Jonsson, J.: Simplicial Complexes of Graphs. Lecture Notes in Mathematics, vol. 1928. Springer, New York (2008)

14. Jonsson, J.: Exact sequences for the homology of the matching complex (2008, submitted)

15. Karaguezian, D.B.: Homology of complexes of degree one graphs. Ph.D. Thesis, Stanford University (1994)

16. Karaguezian, D.B., Reiner, V., Wachs, M.L.: Matching complexes, bounded degree graph complexes and weight spaces of $G L_{n}$-complexes. J. Algebra 239, 77-92 (2001)

17. Ksontini, R.: Propriétés homotopiques du complexe de Quillen du groupe symétrique. Ph.D. Thesis, Université de Lausanne (2000) 
18. Pilarczyk, P.: Computational Homology Program (2004)

19. Reiner, V., Roberts, J.: Minimal resolutions and homology of chessboard and matching complexes. J. Algebraic Combin. 11, 135-154 (2000)

20. Shareshian, J., Wachs, M.L.: Torsion in the matching and chessboard complexes. Adv. Math. 212(2), 525-570 (2007)

21. Wachs, M.L.: Topology of matching, chessboard and general bounded degree graph complexes. Alg. Universalis 49(4), 345-385 (2003)

22. Ziegler, G.M.: Shellability of chessboard complexes. Israel J. Math. 87, 97-110 (1994) 